unmotivirten Einwänden gegenüber zu einer Luxusproduction von Arbeit genöthigt, die sie zum Zwecke anderweitiger Forschungen weit nützlicher verwenden könnten.

Giessen, im März 1872.

\title{
Complicirter Scheidenafter.
}

\author{
Von \\ Dr. Max Bartels, \\ Assistenzarzt am Diakonissenhause Bethanien in Berlin.
}

(Mit Abbildungen Tafel XI.)

Ueber Dünndarmscheidenfisteln, die abnorme Communication zwischen der Vagina und dem Ileum, meist intra partum entstanden, wurde vor einiger Zeit von $\mathrm{He}$ ine $\mathbf{1}^{\mathbf{1}}$ ) eine ausführliche Arbeit gestiutzt auf eine eigene Beobachtung veröffentlicht. Die in der Literatur zerstreuten analogen Publicationen wurden von ihm gesammelt und einer eingehenden Besprechung unterworfen. Fin hierher gehöriger Fall, complicirter als alle dort zusammengestellten, kam auf der Abtheilung des Herrn Geheimrath Dr. Wilms im Krankenhause Bethanien in Berlin zur Behandlung. Derselbe bot durch die Mannigfaltigkeit der Abnormitäten eine Reihe von Schwierigkeiten dar, die erst nach wiederholten genauen Untersuchungen einen klaren Einblick in die Fïlle der vorliegenden pathologisehen Veränderungen gestatteten. Hierdurch nicht allein, sondern anch durch die verschiedenen operativen Eingriffe, welche bei ihm zur Anwendung kamen, ist der Fall lehrreich und von allgemeinerem Interesse. In möglichster Kürze lasse ich die Beobachtung hier folgen.

Am 28. September 1869 wird die 39 Jahre alte Arbeiterfran Johanne Wundrach aus Strassburg i. d. M. recipirt, welche angiebt, dass sie bei ihrer letzten vor 2 Monaten stattgehabten Entbindung durch Schuld der Hebamme einen Schaden an den Genitalien erlitten habe, in Folge dessen sie weder Koth noch Urin zu halten im Stande sei. Nach genauerer Untersuchung ergiebt sich, dass aus ihrer Schamspalte ein Gebilde hervorhängt, welches man bei

1) Anus praeternaturalis ileo-vaginalis durch Enterotomie und Naht geheilt. Arch. f. klin. Chir. XI, 485. 
oberflächlicher Betrachtung für den stark prolabirten Uterus halten möchte (Taf. XI Fig. 1.) Es ist stellenweise mit Schleim und mit diphteritischen Fetzen bedeckt. Von der Schamspalte bis zu seinem tiefsten Punkt ist die Entfernung $10 \mathrm{Cm}$. Seine grösste Breite beträgt $8 \mathrm{Cm}$. an der Stelle. Nur der unterste Theil der Schamspalte wird von ihm eingenommen, der oberste Theil bleibt unverdeckt.

Die Oberfläche des Gebildes besitzt mancherlei Unebenheiten and Hervorragungen, welche oft durch narbige Massen von einander getrennt sind. Durch einen schmalen, unregelmässigen Längsstreifen mit glatter Oberfläche, ähnlich der Lippenschleimhaut, wird das Gebilde in eine obere ventrale und eine untere dorsale Abtheilung geschieden.

Der in die Vagina eingeführte Finger constatirt, dass in einer Höhe von etwa $5-6 \mathrm{Cm}$, die Scheidenschleimhant sich nach unten umschlägt und auf die vorgefallenen Theile übergeht. $\mathrm{Nux}$ in der $\mathrm{Me}-$ dianlinie oberhalb des Vorfalls befindet sich ein Defect in dieser Umschlagslinie, durch den der Finger weiter aufwärts die runzlige Schleimhaut der Harnblase, respective einen durch die Urethra eingeführten Katheter fühlt. Ausser dem complicirten Vorfall besitzt die Patientin also noch eine Blasenscheidenfistel von etwa $4 \mathrm{Cm}$. im Durchmesser.

Auf den prolabirten Massen finden sich mehrere Oeffnungen $\mathbf{1}$ ). Die oberste von ihnen liegt anf der Centralseite des Prolapsus dicht an der Schamspalte (Fig. 2 a). Sie ist horizontal gestellt, etwa $2 \mathrm{Cm}$. breit, schlitzförmig. Der Zeigefinger lässt sich bequem in dieselbe einführen und gelangt nach oben in einen überall gleichweiten, mit Schleimhaut ausgekleideten Kanal, dessen Ende man nicht abreichen kann. Aus dieser Oeffnung gingen der Kranken noch während der ersten Zeit ihres Hospitalaufenthalts die Faecalmassen $a b$, während sie aus dem After nur farblosen Schleim, aber schon seit langer Zeit keinen Koth mehr verlor. Führt man zu gleicher Zeit einen Finger in den After, so fuihlt man zwischen dem Mastdarm und dem eben erwähnten Kanal eine ziemlich dicke Scheidewand.

Zwei andere sehr deudich markirte Offnungen liegen ebenfalls auf der Centralseite etwa $21 / 2 \mathrm{Cm}$. von einander entfernt (Fig. 2 b. c.). Sie sind der Anfang und das Ende eines kurzen Kanals, mit sammtartiger Schleimhant ausgekleidet und für den Zeigefinger bequem durchgängig. Eine tiefe, horizontale Narbe bildet eine Schlucht zwischen den beiden Oeffnungen. Etwa einen Monat nach der Aufnahme zerfiel sie, so dass der kurze Kanal hier eine dritte Oeffnung bekam, welche ihn ungefähr halbirte (Fig. 2 d).

Die dorsale Abtheilung des Vorfalls endet nach unten mit einer birnförmigen Spitze, auf der sich ebenfalls eine Oeffnung (Fig. 2 f.) befindet. Hinter dieser Spitze drängt sich aus dem untersten Winkel der Schamspalte eine mit rosiger Schleimhaut bekleidete Erhöhung

1) Der schematische Sagittaldurchschnitt des Vorfalles Fig. 2 wird das Orientiren erleichtern. 
fig. 1.

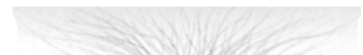


hervor, von der Form und Grösse eines gewöhnlichen Apfels. An seiner tiefsten Stelle hat sie eine grosse Oeffnung (Fig. 2 g.), welche den Zeigefinger bequem passiren lässt. Diese führt in einen Kanal, der etwa $4 \mathrm{Cm}$. aufwärts verläuft; dann biegt er in einen kurzen, engen Kanal mit dicken Wandungen um, welcher die birnförmige Spitze durchbohrt und in der auf ihr befindlichen Oeffnung mündet (Fig. 2 f.).

An der Austrittsstelle des Vorfalls, auf der Dorsalseite, dicht an der Schamspalte gleitet der untersuchende Finger durch eine breite, schlitzförmige Oeffnnng (Fig. 2 h.), welche die Wurzel des Vorfalls wie ein Halbring umgiebt, in einen aufwärtssteigenden Kanal. Ein zugleich in den Mastdarm eingeführter Finger fühlt in einer Höhe von mehr als $8 \mathrm{Cm}$., kaum erreichbar, eine Oeffnung (Fig. $2 \mathrm{~m}$ ) an der vorderen Darmwand. Es ist dieses der Anfang eines nach unten führenden Kanals. Bemüht man sich, mit der Fingerkuppe in die Oeffnung zu dringen, indem man die vordere Mastdarmwand ein wenig herabzerrt, so gelingt es, den Finger etwas in diesen Kanal vorzuschieben. - Man trifft dann auf den in den eben erwähnten aufsteigenden Kanal eingeführten Finger. Somit überzeugt man sich, dass die Oeffnung an der vorderen Mastdarmwand der Anfang, die Oeffnung (Fig. $2 \mathrm{~h}$ ) auf der Dorsalseite des Vorfalls dicht an der Schamspalte das Ende dieses Kanals ist. Durch ihn ging seit der ersten genaneren Untersuchung die Defäcation vor sich, während aus dem After nach wie vor nur Schleimmassen zu Tage traten.

Recapituliren wir in Kürze diesen complicirten Befund, so ergiebt sich, dass der Vorfall von vier Kanälen durchzogen wird, deren einer die oberste Ventralabtheilung (Oeffnung a), ein anderer die unterste Dorsalabtheilung (Oeffaung h) passirt. Beide dienen der Defäcation. Die beiden iubrigen (b. d. c. und $f$ g.) durchsetzen in kurzem, bogenförmigen Laufe die Spitzen des Vorfalles und scheinen keine besondere Funktion zu besitzen.

Für die Beurtheilung des Falles war es von der grössten Wichtigkeit, ïber Lage und Verhalten des Uterus ins Klare zu kommen. An eine operative Behandlung der verschiedenen Kothfisteln konnte nicht gedacht werden, bevor man nicht das ganze prolabirte Gebilde entfernte und auf diese Weise einen einfachen, auf ebener Basis sitzenden Scheidenafter herstellte. Auch die Operation der Blasenscheidenfistel wurde durch den Vorfall unmöglich gemacht, da derselbe die Vagina fast vollständig verlegte. Bei der Form und dem Aussehen des Prolapsus war aber die Möglichkeit nicht von der Hand zu weisen, dass auch der Uterus sich in demselben befände. Man konnte daher nicht ohne Weiteres die Abtragung des Vorfalls vornehmen.

Eine erneute Untersnchung in der Rïckenlage mit möglichst weit geöffneter Vagina ergiebt an der Uebergangsfalte der Vaginalwand auf das prolabirte Gebilde eine spaltförmige, erbsengrosse Oeffnung, welche für eine starke Sonde bequem durchgängig ist. Mit dem durch die Fistel in die Harnblase eingeführten Finger lässt sich der Uterus von normaler Form und Grösse durchfühlen. Die Krüm- 
mung, welche die eingeführte Sonde machen muss, und die Tiefe bis zu der sie sich einfïhren lässt, bestätigen diesen Befund. Die spaltförmige Oeffnung ist das Orificium uteri externum.

Die über diesen Fall gesammelten anamnestischen Daten, die ich theilweise der Freundlichkeit des Herrn Dr. Everth in Strassburg verdanke, ergeben in Kürze folgendes: Die bisher kräftige und gesunde Frau hat ohne Kunsthïlfe 9 Kinder geboren, theils in Kopf-, theils in Beckenendelagen. - Im Juni 1869 erwartete sie ihre zehnte Entbindung. Die Geburt ging sehr langsam vor sich. Die Kreissende hatte viel gelitten und verliess über 12 Stunden nach Abfluss des Fruchtwassers in Verzweiflung das Haus, um sich durch Ertränken das Leben zu nehmen. Ihr Mann hielt sie rechtzeitig zurück, liess aber auf Zureden der Hebamme noch 24 Stunden vergehen, bevor er die Hülfe des Herm Dr. Everth requirirte. Dieser fand das Kind in dritter Schädellage und extrahirte dasselbe mit der Zange ohne Mühe und ohne Schmerzen für die nicht chloroformirte Kreissende. Es war ein kräftiger scheintodter Knabe, welcher nach 12 Stunden starb.

Während der Arzt mit den Wiederbelebungsversuchen beschäftigt war, ging die Hebamme mit der Hand ein, um die Placenta zu entwickeln und zerrte Darmschlingen herab, die sie für die Nachgeburt hielt. Ausser dem oberen Ende des Rectum war eine Dünndarmsehlinge mit ihrem langgezerrten Mesenterium herausgerissen. Die Därme wurden reponirt, die Kranke mit grossen Dosen Opium behandelt. Am Tage nach der Entbindung stand die Frau auf, um sich das Bett machen zu lassen. Sie musste dabei eine Zeit lang aufrecht stehen und stieg dann in das Bett zurück. Hierbei ging die Nachgeburt ab. Als sie sich eben gelegt hatte fühlte sie etwas aus der Schamspalte hervortreten; es waren die Därme von nevem vorgefallen. Mehrfache erneute Repositionsversuche blieben erfolglos; die Därme prolabirten immer wieder. Endlich wurden die Darmschlingen brandig und stiessen sich ab (angeblich melrere Fuss).

Der Prolapsus besteht also aus den Stümpfen der vorgefallenen und grösstentheils brandig abgestossenen Darmschlingen. Dieselben sind durch chronische Entzündung verdickt und unter einander verschmolzen. Zugleich sind sie mit dem. Rändern des Risses im hinteren Scheidengewölbe, durch den sie hervorgefallen waren, eine innige Verwachsung eingegangen. Bei der Exfoliation der brandigen Schlingen musste jede derselben auf dem Vorfall zwei Oeffntngen zurücklassen. Dem steten Druck der Bauchpresse ausgesetzt, welcher durch keinen Sehliessmuskel gemindert wurde, mussten sich die Darmschlingen nach und nach mit ihrer Schleimhaut nach aussen stïlpen, invertiren, wie wir das zuweilen auch nach brandigem Hernien, regelmässig bei denjenigen Bildungshemmungen sehen, bei denen $\mathrm{Ab}$ theilungen des Darmkanals auf der vorderen Bauchwand münden. (Offenbleiben des Ductus omphalo-meseraicus, Bauchblasengenitalspalte). Es kann daher nicht überraschen, den Vorfall grösstentheils mit sammtartiger Schleimhaut bekleidet zu sehen. Es ist dies eben 
die durch Inversion nach aussen gekehrte, jetzt aber theilweise degenerirte Darmschleimhaut.

Die Lage des Uterus an normaler Stelle war constatirt worden. Man konnte somit, sicher, dass der Vorfall keine edleren Organe in sich einschloss, an die operative Entfernung desselben gehen. Die Vagina wurde durch nasse Compressen geschützt, der Prolapsus mit einer nassen Compresse gefast und nun möglichst dicht an seiner Wurzel mit beilförmigen, eigens hierzu construirten Glüheisen abgeschnitten. Die Blutung aus mehreren grösseren und kleineren Arterien, jedenfalls wohl Aesten der Art. meseraica superior ward ebenfalls durch Applikation von Glüheisen gestillt (19. Nov. 1869), Dieser operative Eingriff ging ganz ohne Reaction vorüber.

Ein Durchschnitt durch den amputirten Prolapsus (Fig. 3) zeigt, dass sein ganzer Kern aus Fettgewebsmassen besteht, die durch starke, Gefässe tragende Bindegewebszüge in unregelmässige Läppchen zerfallen. Der Schnitt hat zugleich die Dorsalabtheilung in der Längsaxe getroffen. Man sieht, dass die sammtartige Oberfläche (Fig. $3 \mathrm{~h}$ ) etwa eine Linie im Durchmesser besitzt. An sie stösst eine Zone (Fig. 3 p) von 5-7 Mm. Dicke, welche durch ganz feine, radiär gestellte Bindegewebszüge in lauter einzelne kleine $\mathrm{Ab}$ theilungen getheilt wird. Zwischen diesen Radiärzïgen wölbt sich ein hyalines Gewebe hervor, welches in Form und Farbe an das Fleisch einer durehschnittenen Citrone erinnert. Diese Radiärzone begrenzt nach innen ein etwa $3 \mathrm{Mm}$. breiter Streifen von derbem Bindegewebe (Fig. 3 u), an den sich dann das oben erwähnte Fettgewebe anschliesst (Fig. 3 o).

Ein Durchschnitt durch die birnförmige Spitze zeigt ein ähnliches Bild, nur in noch schönerem Maasse. Die oberflächliche Schicht ist fast ebenso, wie vorher gestaltet. Die Radiärzone schliesst sich fast zu einem Kreise, in dessen Mitte die etwas verbreiterte Bindegewebszone liegt. Der Kern aus Fettgewebe fehlt hier. Die einzelnen radiären Abtheilungen, welche sich noch stärker hervorwölben, als auf der vorigen Schnittfläche; stehen jede mit ihrer äussersten und innersten Spitze in einem tieferen Niveau, als mit ihrer Mitte, so dass diese stark prominirt. Es entsteht auf diese Weise die Form eines Turbans. Durch die hyaline, glänzende Beschaffenheit der einzelnen Radiärabtheilungen und ịre grosse Regelmässigkeit wird ein überaus zartes Bild hervorgerufen.

Die mikroskopische Untersuchung erweist die Radiärzone als aus organischen Muskelfasern bestehend. Es ist die colossal hyperplastische Muscularis des Darmes. Die oberflächliche, sammetweiche Schicht wird durch ihren Reichthum an Drüsen als die ebenfalls hyperplastische Darmschleimhaut charakterisirt. Den Bindegewebsstreifen medianwärts von der Radiärzone wird man danach als Reste der Serosa, das starke Fettgewebe als degenerirtes Mesenterium zu deuten haben.

Siebzehn Tage nach der Operation (6. December) wurde die Kranke wieder untersucht. Man konnte erwarten, an der hinteren Vaginalwand eine Unebenheit vorzufinden, als Rest des abgetragenen 
Vorfalls. Es hatte sich aber die Vagina vollständig geglättet und man fand nur nahe dem Orificium nteri externum eine über $\mathrm{Zwei-}$ groschensück grosse Oeffnung, den Scheidenafter. In dieser Oeffnung mussten mindestens zwei Darmabtheilungen zusammenkommen, nämlich erstens das Rectum, zweitens diejenigen Partien des Ileum, iiber deren ferneren Verlauf man nichts Bestimmtes angeben konnte (Fig. 2 a). Dieses Stück des Dünndarm ist jedenfalls sein oberes Ende. Der Scheidenafter erschien aber nur als einfache Oeffnung, man konnte nichts von Scheidewänden in ihm bemerken. Durch ihn war nach wie vor die Defäcation vor sich gegangen; Stuhlgang auf normalem Wege war bis jetzt noch nicht dagewesen. Da man den Mastdarm bei der Untersuchung aber mit Scybalis erfiullt fand, so wurden jetzt täglich Clysmata angewendet, die ihren Zweck vollkommen erreichten.

Im Januar 1870 wurde der Versuch gemacht, den Schoidenafter, der schon von einem schönen Narbenring umgeben war, durch wiederholte Application des Ferrum candens zum Schluss zu bringen. So lange der Brandschorf lag, war die Defäcation in schönster Ordnung, die Vagina blieb vollkommen rein. Sobald aber die Eschara sich abstiess, gingen auch wieder bei jedem Stuhlgang Kothmassen durch die Scheide ab. Der Scheidenafter verkleinerte sich aber durch Narbencontraction soweit, dass man nur noch die Kuppe des kleinen Fingers einführen konnte.

Im Februar, acht Monate nach der Entbindung trat zum ersten Male auf zwei Tage die Regel, begleitet von unangenehmen Empfindungen im Leibe, ein.

Nach einer Koprostase entleerte sich Ende Februar ein dicker Stuhlgang per vaginam; durch den After ging fast gar nichts $a b$. Dér durch das Glüheisen erzielte Erfolg war also vollkommen wieder aufgehoben. Herr Geheimrath Wilms entschloss sich daher, den Scheidenafter auf blutigem Wege zu schliessen. Auch die Blasen= scheidenfistel harrte noch ihrer Operation und es entstand nun die wichtige Frage, in welcher Reihenfolge man die Operationen vornehmen sollte. Schloss man die Blasenscheidenfistel zuerst, so waren ihre adaptirten Ränder durch den Scheidenafter dauernd mit Fäcalmassen bedeckt; liess man dem Scheidenafter den Vorrang, so wurde die frische Operationswunde von dem perpetuirlich aus der Blasenscheidenfistel aussickerden Urin macerirt. Diese beiden Hindernisse der Heilung räumte man aus dem Wege, wenn man beide abnorme Oeffnungen in einer Sitzung' operirte.

Um durch den etwaigen Eintritt der Menstruation nicht gestört zu werden, wartete man erst 5 Wochen nach der ersten Regel ab Als sich aber in dieser Zeit nichts zeigte, schritt Herr Geheimrath ${ }^{\circ}$ Wilms am 8. März zur Operation. Vorher wurde die Kranke noch einmal genau untersucht.

An der hinteren Wand der Vagina erblickt man etwa $1^{1 / 2} \mathrm{Cm}$. vom Orificium uteri in der Mittellinie den sechsergrossen, trichterförmigen Scheidenafter, umschlossen von lividen, granulirten Rän- 
dern. Das Orificium ist : spaltförmig, quer im Niveau der Scheide liegend. Eine Portio vaginalis existirt nicht, ebenso wenig ein vorderes und hinteres Scheidengewölbe. Die zwischen dem Muttermund und dem Scheidenafter liegende Vaginalschleimhaut ist völlig normal und zeigt keine Spur einer Narbe.

Die vordere Wand der Scheide endet nach oben mit einem bogenförmigen, scharfen Rande, etwa $2 \mathrm{Cm}$. vom Orificium ateri entfernt. Dieser zwischen dem Muttermunde und dem freien Rande der Scheidenschleimhaut liegende Raum ist die Blasenscheidenfistel und wird ausgefüllt durch die hervorgewölbte Schleimhaut der Harnblase.

Es wurde nun zuerst der Scheidenafter in seinem ganzen Umkreise breit trichterförmig auf das genaueste angefrischt. Als zweiter Act der Operation wird die trichterförmige Anfrischung der Blasenscheidenfistel vorgenommen. Mit Silbernähten und dazwischen gelegten Seidensuturen werden darauf die Wundflächen des Scheidenafters sorgfältig adaptirt und möglichst fest geschlossen, so dass eine transversale Narbe gebildet wird. Die Blasenscheidenfistel wird ebenfalls in querer Richtung durch Silber - und Seidennähte geschlossen, und nachdem man sich durch Einspritzen in die Harnblase überzeugt hat, dass der Schluss der Blasenseheidenfistel auf allen Punkten ein vollkommener ist, ist die Operation beendet. Ein wenig Charpie wird in die Vagina gelegt, um die beiden sich gegenüberliegenden Wunden vor dem Reize der mässig kurz abgeschnittenen Silberdrähte zu schïtzen.

Die Nachbehandlung war die in Bethanien bei Blasenscheidenfisteln gebräuchliche. Patientin wird mit mässig gelkrümmten durch dreieckige Kissen unterstützten Knieen auf den Rücken gelagert, auf 8-14 Tage verstopft gehalten bei Verabreichung von nur flüssiger Nahrung. In die Harnblase wird ein elastischer Katheter eingelegt, der perpetuirlich den Urin abfliessen lässt.

- Die Operationen verliefen ebenso wie die frühere ohne Reaction, nur dass am Tage nachher unter mässigen peritonitischen Reizungen die fünf Wochen vergeblich erwartete Menstruation eintrat. Vier Tage nach der Operation gehen Blähungen durch die Scheide ab. Am 20. Tage zeigt sich die Blasenscheidenfistel vollständig geheilt und vernarbt. Bei dem Scheidenafter jedoch ist grösstentheils die Verschmelzung der Wundfächen ausgeblieben. Er ist nur etwas verkleinert und bildet jetzt noch einen erbsengrossen von schwammigem Gewebe umgebenen Defect.

Bis zum Anfange April nahm die Verkleinerung des Scheidenafters noch bis zur Grösse einer Linse zu, dann blieb er unverändet. Deswegen und weil noch immer viel Stuhlgang seinen Weg durch die Scheide nahm, führte Herr Geheimrathe Wilm's am 20. April die Operation in der oben beschriebenen Weise noch ein Mal aus. Wäbrend aber damals die transverselle Obliteration angestrebt wurde, wurde dieses Mal die Wunde in der Längsrichtung geschlossen.

Am Tage darauf klagt die Kranke über unangenehme Empfindungen im Leibe. Am zweiten Tage tritt die Regel ein, sechs 
Wochen nach der letzten Menstruation and ebenso wie diese direct nach der Operation. Es bestätigte sich hier wieder eine Beobachtung, die wir schon mehrmals zu machen Gelegenheit hatten, dass nämlich blutige Operationen im Bereiche der Scheide oder der äusseren Genitalien ein sehr wirksames Emmenagogum sind. Es ist uns mehrfach vorgekommen, dass wir bei Patientinnen, denen derartige Operationen bevorstanden (Blasenscheidenfisteln, Harnröhrenfisteln, alte Dammrisse) längere Zeit vergeblich den Eintritt der Menstruation abwarteten; kaum aber war die Operation ansgeführt, so stellte sich auch am ersten oder zweiten Tage danach die Regel ein.

Schon am Tage nach der Operation waren Blähungen durch die Vagina abgegangen. Einen Tag später und eben so auch die folgenden Tage traten auch feste Fäcalmassen durch die Scheide aus. Es kunnte also kein Zweifel darüber bestehen, dass eine Heilung: des Scheidenafters auch dieses Mal nicht eingetreten sei. Anfang Mai, nach Entfernung sämmtlicher Nähte, wird daher wieder zur Application des Glüheisens geschritten, und später die Wunde mit Tinet. cantharidum behandelt. Stuhlgang hat die Kranke täglich, aber durch die Vaginas per Anum geht so gut wie nichts.

Am 7. Mai, zwei und eine halbe Woche nach der Operation, ist die Narbe der Blasenscheidenfistel nur noch als eine ganz feine Zickzacklinie sichtbar. Das Orificium uteri ist ein wenig erodirt. Der Scheidenafter ist von einem ziemlich festen, callösen Rande umgeben und lässt bei Anwendung einiger Gewalt, wie das zur genauen Untersuchung nöthig war, die Kuppe des Zeigefingers durchtreten und zwar in das Rectum. Versuche, in das obere Darmende zu kommen, waren vergeblich. Bei allen diesen Versuchen drang die Fingerspitze jedes Mal über den ziemlich verdickten Rand fort in den Mastdarm. Ein in den Anus hoch hinaufgefïhrter Finger war nicht im Stande, die obere Fortsetzung des Rectum aufzufinden. Man fühlte die Mastdarmwände ein Gewölbe bilden, das nach oben vorn allein einen Ausgang gestattete. Dieser Ausgang führte in die Scheide.

Das Pfingstfest stand vor der Thür. Die Patientin (die ganze Zeit im Krankenhause gratis verpflegt) wurde von einer unbezwinglichen Sehnsucht nach ihrer Familie befallen, die sie nun 8 Monate nicht gesehen hatte. Sie verlangte trotz eindringlicher Gegenvorstellungen ihre Entlassung. Am 14. Mai verliess sie, zwar geheilt von ihrer Blasenscheidenfistel, aber mit noch bestehendem Scheidenafter. das Hospital.

Die letzte genaue Digitaluntersuchung hatte aber für die Kranke den günstigen Erfolg gehabt, dass jetzt die Defäcationen wieder auf normalem. Wege vor sich gingen und dabei jedes Mal nur sehr wenig Koth durch den widernatürlichen After in die Scheide trat.

Nach circa einem halben Jahre meldete mir die Patientin, dass sie, obgleich dex Stuhlgang grösstentheils wieder dureh die Vagina entleert würde, sich in gutem Wohlsein befände und auch bei gutem Appetit alle Speisen vertrage. Ein erneuter Vorfall des Dünndarmes durch den Scheidenafter war bisher nicht eingetreten. 
Dieser merkwïrdige Fall bietet noch mancherlei Räthselhaftes and Unklares. Wie verhalten sich hier die verschiedenen Abtheilungen des Tractus intestinalis zu einander? Welche Theile sind in Function, welche sind für den Diэnst des Organismus verloren gegangen? Es fehlen bestimmt grosse Stücke des Ileum; sie gingen brandig zu Grunde, das ergab die Anamnese. In der Vagina fanden sich oft halbverdaute Speisereste und dünnflüssiger Koth oder besser Chymus, welcher bestimmt aus dem oberen Ende des Dünndarms stammte: Das ist natürlich, denn dieses obere Ende (Fig. 2 a) mündet in den Scheidenafter.

Es kann auch nicht überraschen, dass Stoffe, die dem oberen Dünndarmabschnitt entstammen, durch das Rectum aus dem After treten, denn wir sahen ja, dass in dem Scheidenafter Dünndarm und Mastdarm mit einander communicirten. Was ist nun aus dem ganzen Dickdarm geworden? Ist er ausser Function gesetzt und collabirt, wie das bei nicht functionirenden Darmpartien die Regel ist? Hiergegen spricht erstens die ganz normale Configuration des Bauches und zweitens der reichliche Abgang von Koth, wie er nur im Dickdarm sich bildet.

Dieser Koth ging, wie wir sahen, theils durch den natürlichen, theils durch den Scheidenafter ab. Er stammt ans dem Colon und tritt in den Mastdarm, es ist also die Communication zwischen diesen beiden Darmpartien erhalten. Wie ist es aber dem Dickdarm möglich, Fäces zu liefern? Wie gelangen die Speisen, die doch jedenfalls die oberen Dünndarmpartien passiren müssen, zu ihm? Es muss hiernach auch zweifellos eine Verbindung zwischen dem Colon und dem oberen Stück des Ileum vorhauden sein, das, wie erwähnt, in den Scheidenafter ausläuft. Wo diese Verbindung sich befindet und auf welche Weise sie möglich wurde, das erklären zu wollen, würde uns auf ein unfruchtbares Gebiet der Hypothesen führen. Nur eine genaue Obduction wird im Stande sein, sichere Aufklärung hierïber zu verschaffen.

\section{Erklärung der Abbildungen.}

Taf. XI. Fig. 1. Ansicht des Vorfalls.

Fig. 2. Schematischer Sagittaldurchschnitt durch den Vorfall. $a$ und $h$ Mündungen der Koth führenden Kanäle; $b$ d $c$ und $f g$ functionslose Kanäle; $n$ normaler After; $m$ Oeffnung in der vorderen Wand des Mastdarmes.

Fig. 3. Sehnittfläche des amputirten Vorfalles. t Hypertrophische Schleimhaut; $\mathrm{p}$ hypertrophische Muscularis; u Bindegewebsstreifen; o Kern von gelapptem Fettgewebe. 